\title{
Intervention Ergonomique Capacitante : bilan des connaissances actuelles et perspectives de développement
}

Flore Barcellini

\section{(Q) OpenEdition}

\section{Journals}

Édition électronique

URL : http://journals.openedition.org/activites/3041

DOI : 10.4000/activites.3041

ISSN : $1765-2723$

Éditeur

ARPACT - Association Recherches et Pratiques sur les ACTivités

Référence électronique

Flore Barcellini, « Intervention Ergonomique Capacitante : bilan des connaissances actuelles et perspectives de développement », Activités [En ligne], 14-2 | 2017, mis en ligne le 15 octobre 2017, consulté le 20 avril 2019. URL : http://journals.openedition.org/activites/3041 ; DOI : 10.4000/ activites.3041

Ce document a été généré automatiquement le 20 avril 2019.

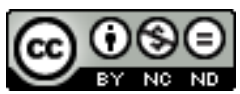

Activités est mis à disposition selon les termes de la licence Creative Commons Attribution - Pas d'Utilisation Commerciale - Pas de Modification 4.0 International. 


\title{
Intervention Ergonomique Capacitante : bilan des connaissances actuelles et perspectives de développement
}

\author{
Flore Barcellini
}

\section{NOTE DE L'AUTEUR}

L'auteure tient à remercier l'ensemble des participants à ce numéro spécial pour la qualité de leurs articles, ainsi que les éditrices (Justine Arnoud et Maria Sol Perez Toralla) et l'éditeur (Alexandre Morais) de ce numéro spécial.

1 Ce numéro spécial s'est donné pour objectif de répondre à une question essentielle pour le développement de l'ergonomie: peut-on parler d'intervention ergonomique capacitante et dans ce cas quelles en seraient les visées et caractéristiques? Cette question apparaît essentielle, car, d'une part, elle permet de renouveler les objectifs des interventions ergonomiques en assumant leur visée « développementale » qui n'est plus uniquement vue comme un effet «induit» de l'intervention, mais bien un effet recherché. D'autre part, cette question assume le rôle des actions ergonomiques dans la conception des organisations, comme substrat essentiel au développement des activités, à la performance et à la construction de la santé des opérateur.trice.s. Elle renforce alors l'ergonomie comme une des disciplines contributrices de démarches de conduite de changements organisationnels. Assumer cette vision développementale implique de répondre le plus finement possible à plusieurs questions : qu'est-ce qu'une intervention ergonomique capacitante, quelles en sont les visées, les éléments structurants? Quels dispositifs spécifiques doivent être conçus et intégrés aux interventions, avec quelle visée développementale? Quelles transformations de l'activité de l'ergonome? Quelles conséquences pour le développement de la discipline, son enseignement, le 
positionnement de l'ergonomie de l'activité au niveau international, son positionnement vis-à-vis de disciplines connexes?

Les articles de ce numéro spécial font considérablement progresser la discipline dans la réponse à ces questions, même si des défis demeurent, comme le soulignent Arnoud et Perez Toralla dans leur introduction. Dans la suite de cette conclusion, nous précisons dans une première section les spécificités des interventions ergonomiques capacitantes et leurs objectifs et éléments structurants. Les sections suivantes détaillent les apports de ce numéro à la compréhension de ces éléments structurants : la genèse et les spécificités de la construction sociale des interventions ergonomiques capacitantes, les dispositifs d'actions développementaux déployés dans ces interventions et enfin les dispositifs de généralisation et de pérennisation. Nous concluons sur les évolutions de l'activité de l'ergonome sous-tendues par le développement des interventions ergonomiques capacitantes. Enfin, nous définissons un ensemble des perspectives de recherche, d'enseignement et de développement de la discipline.

\section{Intervention ergonomique capacitante et développement de la capacité des organisations à se transformer en prenant en compte le travail}

\subsection{Les interventions ergonomiques ne sont-elles pas toutes capacitantes?}

Attaquons-nous d'emblée à une question fréquemment posée: les interventions ergonomiques ne sont-elles pas toutes « capacitantes »?

Une analyse des définitions proposées par les différent.e.s auteur.e.s de ce numéro - c'està-dire la manière dont ces auteur.e.s s'approprient ce concept - permet de dégager plusieurs spécificités de ce que sont, pour eux.elles, des interventions ergonomiques capacitantes.

Dans ces définitions, le développement des individus, des collectifs et de l'organisation est posé comme objectif de l'intervention, ce qui a trois conséquences.

Tout d'abord, le développement (des individus, des collectifs et des organisations) n'est pas uniquement un effet induit de l'intervention ergonomique, mais bien sa finalité, qui est alors explicitée et négociée avec les demandeurs. C'est le cas de toutes les interventions - sans exception - de ce numéro.

7 Par ailleurs, le développement (des individus, des collectifs et des organisations) implique que l'intervention ergonomique capacitante vise la conception $d$ '« organisation capacitante », définie par deux caractéristiques principales :

- D'une part, une organisation capacitante est une organisation ${ }^{1}$ capable de se transformer - de se développer - en créant les conditions du débat des règles organisationnelles et de leurs évolutions (p. ex. à travers des espaces de discussion sur le travail, cf. infra). Arnoud et Perez Toralla soulignent que cette organisation « se prête à l'adaptation d'elle-même » - à condition que l'analyse et la discussion de ces évolutions d'usage soient soutenues, c'est-à-dire que les règles organisationnelles soient débattables. Carta et Falzon (ce dossier) voient ce renouvellement comme "la mise en marche de mécanismes autopoḯtiques (Carta, Falzon, \& Re, 2015), qui permettent à l'organisation de se 
renouveler de manière autonome ». La vision des organisations du travail portée ici est donc bien celle d'une organisation "instrumentalisable", dont les règles peuvent être remaniées - reconçues - à tous les niveaux d'une organisation (Rocha, Mollo, \& Daniellou, ce dossier). L'organisation n'est donc pas vue comme une structure figée et immuable tel un artefact conçu qui ne pourrait évoluer. Cette vision d'une organisation capacitante comme « débattable et instrumentalisable » implique que les organisations : (1) mettent en place des espaces de « débats et confrontation de points de vue " sur « les conflits de critères, de buts, de logiques sur le travail $\aleph^{2}$, et plus largement (2) soutiennent l'autonomie et l'activité réflexive des opérateur.trice.s de tout niveau hiérarchique tant au niveau individuel que collectif (Arnoud, 2013 ; Mollo \& Nascimento, 2013) (3) permettent la prise en compte des capacités d'innovation des opérateur.trice.s pour qu'elles puissent «être intégrées à la structure (organisationnelle) de telle sorte que la conception se poursuive dans l'usage » (Arnoud \& Falzon, 2013, p. 224). Or, comme le rappelle notamment Bonnefond (ce dossier), les organisations du travail contemporaines n'assument pas «la délibération sur les conflits de critères sur la qualité du travail (Clot, 2010)", ce qui « se retourne contre la santé et l'efficacité ». Une organisation capacitante se doit donc de restaurer « ce dialogue autour du conflit de critères ». Ainsi, la visée de l'intervention capacitante « consiste à concevoir non pas une nouvelle organisation, mais un dispositif d'expérimentation et d'apprentissage, pour susciter et favoriser de nouveaux fonctionnements organisationnels" (Sardas \& Lefevre in Arnoud \& Falzon, 2013).

- D'autre part, une organisation capacitante est une organisation capable de créer les conditions du développement des individus et des collectifs. Carta et Falzon ainsi qu' Arnoud et Perez-Toralla nous rappellent que développements des organisations, des collectifs et des individus sont intriqués : le développement des organisations est soutenu par le développement des individus (p.ex. développement de leurs compétences à prendre en compte le travail dans la transformation des organisations) et des collectifs (p.ex. développement de la capacité à co-élaborer des critères communs de qualité du travail). Mais, le développement des organisations - sous-entendu capacitantes - soutient en retour le développement des individus et des collectifs, notamment à travers des possibilités de conception d'environnements de travail "capacitants", capables de favoriser le développement des compétences, l'autonomie, le pouvoir d'agir des individus et des collectifs (Falzon, 2013). Ainsi, «L'intervention développementale vise à favoriser les apprentissages au cours même de son déroulement (Falzon \& Barcellini, 2014), et à promouvoir la conception de systèmes de travail qui eux-mêmes favoriseront les apprentissages et le développement (Arnoud \& Falzon, 2017; Barcellini, 2015) 》(Carta \& Falzon, ce dossier).

Cet objectif (viser la conception d'une organisation capacitante) renforce le positionnement - certes déjà présent (p. ex. Petit, 2005) - de l'action ergonomique aux niveaux politique, stratégique et organisationnel (Carta et Falzon, ce dossier). Par ailleurs, il renforce la vision de l'intervention ergonomique vue comme un acte pédagogique (Dugué, Petit, \& Daniellou, 2010). Girardot et Forrierre et Carta et Falzon insistent particulièrement sur cette dimension. En effet, l'intervention décrite par Girardot et Forrierre, est construite comme une formation-action à l'analyse et à la mise en débat du travail, qui a été un outil pour le développement des individus, et « $a$ contribué à favoriser leur participation et a mené à une meilleure appropriation de la démarche" (Girardot \& Forrierre, ce dossier). Carta et Falzon ont pensé leur intervention comme un processus d'apprentissage soutenant le développement des individus (Falzon, 2013 ; Carta \& Falzon, ce dossier), à travers la mise en place d'un laboratoire développemental qui combine un dispositif d'intervention et d'apprentissage. Démarche de formation-apprentissage et 
interventions ergonomiques capacitantes sont donc «intimement liées» (Delgoulet \& Vidal-Gomel, 2013; Girardot \& Forrierre, ce dossier). Nous y reviendrons dans la section 3.

Enfin, une intervention ergonomique capacitante est d'emblée pensée pour générer une transformation durable de l'organisation. Celle-ci crée donc les conditions de son propre dépassement (Coutarel \& Petit, 2009) et vise à « organiser l'existant et le futur de sorte qu'ils permettent aux organisations de progresser (Falzon, 2013)» (Carta \& Falzon, ce dossier). Cette durabilité de l'action est au cœur des définitions et il s'agit d'une spécificité majeure des interventions ergonomiques capacitantes qui place la visée de l'action ergonomique dans un "long terme ", " pour s'inscrire à l'échelle de la vie quotidienne de l'organisation » (Casse \& Caroly, ce dossier). Ainsi, une intervention ergonomique capacitante initie une démarche de développement au sein de l'organisation qui continuera à se déployer après cette intervention, comme le décrivent Bonnefond et Carta et Falzon. Initier ce développement est donc un moyen de l'intervention et l'intervention est pensée donc comme un processus d'apprentissage soutenant le développement (Falzon, 2013; Carta \& Falzon, ce dossier). La focalisation des interventions ergonomiques capacitantes ne doivent pas faire oublier une dimension essentielle de ces interventions, elles conservent un objectif productif (Carta \& Falzon, ce dossier) et visent bien la transformation, c'est-àdire la conception ou la co-élaboration, de productions matérielles (p.ex. règles organisationnelles, espace, logiciels...) et immatérielles (p.ex. connaissances de démarches alternatives de conduites du changement (Carta \& Falzon, ce dossier). Cette dimension "transformation-conception" ne doit pas être oubliée: l'intervention ergonomique capacitante reste selon nous une intervention construite comme un processus de conception ou de co-élaboration de connaissances, devant créer les conditions de la mise en œuvre d'activités collaboratives de conception (Barcellini, 2015). Ce n'est qu'à travers la participation concrète des acteurs à ce processus que les apprentissages opèrent et que les acteurs expérimentent les potentialités de la démarche.

\subsection{Objectifs et éléments structurants d'une intervention ergonomique capacitante}

10 Pour répondre à la question initialement posée, nous dirons que les interventions ergonomiques portent en elles les germes de processus développementaux (Barcellini, 2015; Barcellini, Van Belleghem, \& Daniellou, 2013), mais qu'elles ne suffisent pas à soutenir le développement durable d'organisations capacitantes (Casse \& Caroly, ce dossier), si: (1) elles ne prennent pas comme objet de transformation les questions organisationnelles, et si (2) elles n'associent pas aux dispositifs d'intervention « classiques » (p. ex de type de conduite de projet), des dispositifs d'apprentissage et des dispositifs de pérennisation de la démarche.

11 À travers l'analyse de ces interventions, un premier modèle d'intervention ergonomique capacitante peut-être construit.

12 Une intervention ergonomique capacitante porte trois objectifs (Carta \& Falzon, ce dossier) :

- des objectifs productifs de transformations - de conception d'artefacts organisationnels prenant en compte le travail : démarche participative de conduite du changement (Carta \& Falzon, ce dossier), dispositif de REX (Casse \& Caroly, ce dossier), dispositif de débat sur la qualité du travail (Bonnefond, ce dossier), dispositif de débat sur le travail quotidien en lien 
avec des questions de sécurité ou de santé (Rocha, Mollo, \& Daniellou ; Girardot \& Forrierre, ce dossier) ;

- des objectifs constructifs ou développementaux renvoyant aux développements des individus et des collectifs soutenant en retour le développement des organisations. Ces développements sont soutenus par des apprentissages individuels et collectifs: développement de savoirs quant aux modèles de l'humain en situation de travail, au lien santé-développement-performance ; développement de compétences « analytiques collectives » et de la capacité à construire une « intelligibilité mutuelle » des situations (Carta \& Falzon, ce dossier); développement de compétences à conduire une transformation de manière située, participative et collaborative (Carta \& Falzon, ce dossier ; Barcellini, 2015); développement d'une activité de management centrée sur le travail réel et prenant en charge les transformations...;

- et des objectifs autopoïetiques visant la pérennisation des deux premiers renvoyant au développement organisationnel, c'est-à-dire à la capacité des organisations à se développer de manière durable.

13 Plusieurs éléments structurant les interventions ergonomiques capacitantes peuvent ensuite être dégagés. Les interventions décrites dans ce numéro spécial partagent toutes une posture développementale affirmée qui semble avoir été tenue de manière explicite dans la négociation avec les demandeurs. Elles partagent également toutes une approche par l'expérimentation de dispositifs co-conçus, puis déployer à plus grande échelle (généraliser) et pérenniser. Ainsi :

- Une intervention ergonomique capacitante s'appuie sur une construction sociale proche de celle des interventions de type conduite de projet, si ce n'est que: la dimension développementale, notamment du niveau organisationnel, est négociée d'emblée avec les demandeurs; les structures participatives doivent d'emblée chercher à intégrer toute la ligne managériale pour assurer la pérennité des éléments construits par l'intervention (cf. infra).

- Une intervention ergonomique capacitante s'appuie toujours sur un diagnostic ergonomique, mais celui-ci est finalisé par la dimension développementale des interventions, ce qui implique que les fonctions de ce diagnostic changent. Il s'agit toujours pour l'ergonome de se construire une représentation du travail, des ses variabilités, des régulations, etc., l'ergonome conduisant cette démarche se devant de disposer d'une représentation fine du travail pour construire et conduire une intervention, à visée capacitante ou non. Cependant, ce diagnostic n'a plus uniquement pour objectif d'aider à éclairer différemment les questions de santé et de performance au sein d'une organisation, et d'identifier des projets de transformation, mais bien d'identifier également des besoins de développement organisationnel (Carta \& Falzon, ce dossier). Par ailleurs, les potentialités de co-élaboration de ce diagnostic avec les opérateur.trice.s de toute la ligne managériales pour alimenter les apprentissages sont soulignées (cf. infra). Cette co-élaboration du diagnostic est une production nécessaire à la poursuite de l'intervention ergonomique capacitante : le diagnostic est un objet intermédiaire de cette intervention, mais la co-élaboration du diagnostic est également un moyen de cette intervention puisqu'il soutient les apprentissages et le développement.

- Une intervention ergonomique capacitante s'appuie sur la conception et l'expérimentation d'autres dispositifs de mise en visibilité, de mise en débat du travail qui articulent compréhension collective du travail et identification de projets de transformation et proposition de prototypes d'artefacts organisationnels en réponse à ces projets. Un tel dispositif peut renvoyer à la mise en place d'espaces de débats sur le travail outillés par des 
méthodes telles que l'auto-confrontation croisée ou la simulation d'évènements (passés ou à venir). Il peut renvoyer à des dispositifs de conception, tel qu'ils sont plus classiquement travaillés par la discipline. En tant qu'intervention à visée de transformation, l'intervention ergonomique capacitante hérite des propriétés et des méthodes d'une intervention de type conduite de projet.

- Et enfin, une intervention s'appuie sur des dispositifs de généralisation puis de pérennisation des démarches.

14 Nous détaillons dans les suites ce que nous apprennent les différentes interventions sur ces éléments structurants.

\section{Genèse et construction sociale des interventions ergonomiques capacitantes}

Un premier élément structurant des interventions ergonomiques capacitantes porte sur la construction sociale spécifique de ces interventions. Celle-ci est, d'une part, liée à la genèse des interventions et à des reformulations de la demande explicitement tournées vers le développement. D'autre part, la construction sociale s'appuie sur la mise en place de situations de participation effective de différents niveaux hiérarchiques, comme conditions sine qua non de la durabilité des interventions.

\subsection{Genèse des interventions capacitantes}

Dans Barcellini (2015), nous avions proposé la définition d'interventions capacitantes dans un cadre spécifique, celui de la conduite du changement - Interventions Capacitantes en Conduite du Changement (I3C). L'idée était de positionner explicitement l'ergonomie comme contributrice à la conduite des changements dans les organisations, à la suite des interventions capacitantes princeps d'Arnoud (2013) et Raspaud (2014) qui se déroulaient dans ce cadre (resp. création d'un centre de service RH partagé dans une grande entreprise de transport et introduction de la chirurgie ambulatoire dans un $\mathrm{CHU}$ ). Or, les portes d'entrée des interventions présentées dans ce numéro dépassent largement ce cadre : à l'exception de Carta et Falzon, qui interviennent d'emblée dans un contexte de changement organisationnel (re-internalisation de la maintenance de lignes de métro), les autres interventions s'inscrivent dans des demandes diverses, liées à des questions de santé - le lien santé et qualité du travail chez Bonnefond ou la prévention de la pénibilité du travail et des TMS chez des aides-soignantes chez Girardot et Forriere - ou de gestion de la sécurité chez des techniciens-gaziers pour Rocha et al.ou des patrouilleurs autoroutiers chez Casse et Caroly.

17 Pour finir sur ces éléments contextuels, à l'exception de l'intervention décrite par Girardot et Forrierre, toutes les interventions décrites s'inscrivent dans des dispositifs de recherche. Ceci ne doit pas être interprété comme le fait que ces interventions ne sont possibles que dans ce cadre, mais plus la volonté des chercheurs et enseignantschercheurs d'expérimenter « in vivo » ce que peuvent être des interventions capacitantes et de contribuer à développer les propositions actuelles de l'ergonomie constructive. 


\subsection{Une reformulation des demandes ancrée explicitement dans une visée développementale et s'inscrivant dans une diversité de contexte}

18 Les interventions décrites dans ce numéro spécial partagent toutes une posture développementale affirmée. Carta et Falzon soulignent qu'un discours orienté d'emblée «sous le registre du développement et de l'organisation » et incluant « les enjeux de durabilité de l'intervention afin d'éviter que l'intervention ne soit qu'une parenthèse éphémère dans la vie de l'organisation » (Carta \& Falzon, ce dossier) peut selon eux « aider à rassembler » (ibid., p. 5) les acteurs des sphères managériales et décisionnelles.

19 Cette posture développementale apparaît comme tenue de manière explicite dans la négociation avec les demandeurs des interventions proposées. Le processus et le résultat de cette négociation constituent ainsi la genèse des processus de développement et de la dimension capacitante de l'intervention. Bonnefond illustre très bien ce mouvement de négociation : la demande initiale était portée par la direction d'une usine automobile qui souhaitait un arbitrage des intervenants concernant les désaccords entre direction et organisations syndicales en termes de santé et de qualité des situations de travail. Cette demande a été retravaillée pour aboutir à une proposition d'expérimentation, à plusieurs niveaux hiérarchiques (des opérateur.trice.s à la direction de l'usine, puis du groupe), portant sur la mise en place d'un dispositif dit "de dialogue » autour des critères de qualité du travail "afin de construire avec les opérationnels concernés, des arbitrages organisationnels nouveaux qui soient sources de santé et de performance» (Bonnefond, ce dossier). Girardot et Forrierre décrivent quant à eux que c'est la rencontre entre le contexte de l'intervention - difficultés d'accès direct aux situations de travail (travail à domicile) et temporalité de l'intervention - et la demande initiale "aider nous à aller plus loin pour intégrer une culture de la prévention et à y aller ensemble » qui a été à l'origine de l'intervention proposée - une formation-action à l'analyse du travail et une coconstruction d'actions de transformation. Pour ces auteur.e.s, la dimension capacitante de l'intervention était bien présente dès le début de l'intervention, mais d'abord pensée comme "un élément de régulation méthodologique (pour faire face aux différentes contraintes propres au contexte) » que comme un objectif de l'intervention, objectif qui s'est révélé progressivement au cours de la démarche "en particulier dans l'idée de la pérennisation de la démarche ». Chez Casse et Caroly, ou Rocha et al., on observe également comment des demandes orientées vers la prévention des risques ou la gestion de la sécurité sont renégociées dans une perspective plus développementale, par exemple, le développement d'une approche constructive des dispositifs de retour d'expérience " centrée sur la gestion de la sécurité au quotidien et le travail collectif inter-métiers » chez Casse et Caroly.

\subsection{Construction sociale, participation effective et développement organisationnel durable}

Les interventions présentées dans ce numéro s'appuient toutes sur une construction sociale visant la mise en réseau d'acteurs de tous les niveaux hiérarchiques tout au long de l'intervention, de manière opportuniste (Noyer \& Barcellini, 2014) ou plus planifiée (cf. section 4). L'engagement de toute la ligne managériale (du management de proximité au « top management ») est essentiel, car c'est elle « qui octroiera les ressources et les moyens de 
"dés-empêcher » les managers de réguler le travail » (Rocha et al., ce dossier) et garantira un degré d'autonomie suffisant à la poursuite de la démarche d'intervention, puis la pérennisation de ses résultats.

21 Dans les interventions ergonomiques capacitantes, ces acteurs participent effectivement à la démarche, en expérimentant, avec les intervenants le dispositif d'intervention et ses résultats. C'est cette participation effective qui contribue au développement de l'activité de ces acteurs (Barcellini, 2015 ; Barcellini et al., 2013 ; Béguin, 2003), et qui assure la possibilité de développement durable des organisations (cf. infra). "Effective » est employé ici à dessein pour défendre un modèle "fort » de la participation. En effet, plusieurs auteurs soulignent les risques d'une participation parfois galvaudée dans les entreprises, et donc «jugée comme factice » (p. ex. Bourgeois, 2012, p. 145 ; Perez Toralla, 2013). Par participation effective, nous entendons donc la possibilité réelle de mettre en œuvre une activité de co-élaboration (p.ex. de solutions organisationnelles) à travers une mise en débat « outillée » du travail, mise en débat qui est finalisée par des actions de transformations elles aussi outillées (cf.infra).

22 Ceci implique de concevoir et d'intégrer des dispositifs spécifiques, ensemble de méthodes et de situations d'interactions particulières, permettant de soutenir le développement des individus et des collectifs. Ces ce que nous développons dans la section suivante.

\section{Concevoir et intégrer des dispositifs d'actions développementaux dans les interventions}

Les dispositifs d'action développementaux mis en place s'appuient sur une mise en discussion du travail soutenant des processus réflexifs qui alimentent les processus d'apprentissage et de développement. Nous verrons, dans la première section, que la qualité de cette mise en débat est essentielle à la construction des connaissances nécessaires aux interventions capacitantes. Les sections suivantes décriront les dispositifs d'action mis en place pour soutenir cette mise en débat: dispositifs de formationapprentissages explicites ou dispositifs « outillés » de mise en débat du travail.

\subsection{Discussion et réflexivité sur le travail à visée de transformation}

Dans toutes les interventions présentées, la mise en visibilité du travail est essentielle. Cette mise en visibilité vise à soutenir des processus réflexifs qui alimentent la coélaboration de connaissances autour du travail et de l'organisation puis leurs évolutions. Que les auteur.e.s le revendiquent ou non, il s'agit dans toutes les interventions de créer des espaces-temps outillés par des objets intermédiaires (Vinck, 2009) représentant «le travail » (ou certains de ces aspects) et visant une mise en discussion - un débat (Rocha et al. ; Casse \& Caroly, ce dossier) - un dialogue (Bonnefond, ce dossier) autour des conflits (d'objectifs, de normes, de valeurs, de critères de qualité du travail), des contradictions et des difficultés du travail. Et, c'est bien cette mise en discussion et la tentative de résolution collaborative ${ }^{3}$ de ces conflits, contradictions et des difficultés, qui est source d'apprentissages et de développement. On retrouve ici "le caractère social, situé et dialogique de la construction des savoirs» (Carta \& Falzon, ce dossier), mis en avant par la théorie de l'activité (Vygotsky, 1997) ou le paradigme socio-constructiviste (Doise \& 
Mugny, 1981). L'utilisation de supports représentant le travail et agissant comme des objets intermédiaires permet de mettre en visibilité le travail, d'en faire un objet de réflexion (Pastré, 2005 ; Schön, 1987) «manipulable et discutable » par les participants, soutenant ainsi la confrontation de différents points de vue et interprétations de la situation et ainsi la co-élaboration de sens ou de connaissances (p. ex. explications de telle ou telle difficultés...). Ce sont ces interactions qui sont le moteur des apprentissages, car elles soutiennent les processus de compréhension (p. ex. Dillenbourg et al., 1995). Les interactions argumentatives, notamment, soutiennent des processus de négociation de sens, de re-conceptualisation et de construction de consensus liées à la compréhension des situations discutées (Andriessen, Baker, \& Suthers, 2003 ; Baker, 2009 ; Muller Mirza \& Perret-Clermont, 2009). Il y a alors élaboration de connaissances dans, et par, le dialogue, à travers la gestion des conflits et contradictions qui est un moteur de l'apprentissage (Baker, 2004). Les discussions argumentatives obligent, ainsi, à expliciter ses raisonnements, ce qui déclenche un processus réflexif et une restructuration des connaissances qui soutiennent les apprentissages (Baker, 2004).

C'est cette construction collaborative de connaissances qui porte la genèse de processus développementaux, dans le sens où ces nouvelles connaissances vont soutenir les transformations du travail et des organisations. Il est néanmoins essentiel de préciser que cette « mise en réflexivité » sur le travail ne se suffit pas à elle-même et est finalisée par la transformation de l'organisation et du travail qui doivent être soutenues par l'intervention ergonomique capacitante (Carta \& Falzon, supra).

Plusieurs méthodes sont mobilisées par les auteurs pour mettre en discussion le travail et alimenter les processus réflexifs: co-élaboration de diagnostic, espaces de débats/ discussions sur le travail outillés par des auto-confrontations croisées ou des simulations d'évènements. Dans tous les cas, ce sont bien les propriétés d'apprentissages (objectifs constructifs) et de transformations (objectifs productifs) qui sont mises en avant par les auteur.e.s. Par ailleurs, ces interventions ergonomiques capacitantes incluent une démarche participative de conception organisationnelle, qui s'appuie sur les méthodes plus classiquement utilisées en conception (p. ex. Barcellini et al., 2013; Daniellou, 1992 ; Maline, 1994), que nous ne rappellerons pas ici.

\subsection{Dispositifs de formation-apprentissage : co-élaboration du diagnostic et développement d'une compétence analytique collective}

Deux interventions (Carta \& Falzon; Girardo \& Forrierre, ce dossier) s'appuient sur l'intégration explicite et pensée comme telle de dispositifs de formation-apprentissage ${ }^{4}$ dans une intervention ergonomique ${ }^{5}$, dispositifs présentés comme une des composantes d'une intervention capacitante. "Intégration », car ces auteur.e.s soulignent bien que dispositifs d'apprentissage et intervention à visée de transformation sont indissociables et ne se substituent pas. "Explicite et pensée comme tel », car même si «la part de pédagogie est toujours prépondérante et se déploie dans toutes (...) phases de l'intervention" (Dugué, et al., 2010) : (1) il n'est pas certain que cette dimension soit totalement présente dans les interventions, (2) les modèles des interventions passent souvent sous silence cette dimension. Intégrer de tels dispositifs dans les interventions ne va donc pas de soi et mérite que nous nous y arrêtions. 
Ces interventions permettent de préciser quels peuvent être les types de savoirs et de compétences développés par les dispositifs de formation-apprentissages dans des interventions ergonomiques capacitantes. Tout d'abord, les dispositifs de formationapprentissages conçus s'appuient sur des principes pédagogiques issus de la didactique professionnelle (p. ex. Pastré, 2005) et des modèles d'approches socio-constructivistes des apprentissages des adultes. Il s'agit de faciliter les apprentissages en «faisant faire l'expérience » et en permettant la mise à l'épreuve de nouvelles manières de faire (Carta \& Falzon, ce dossier). Les dispositifs mis en place s'appuient sur différents éléments : l'apport de connaissances situées et le développement de compétences d'analyse collective centrées sur la réalité du travail et des organisations.

partes le « registre de l'expertise ", c'est-à-dire des apports de connaissances "théoriques" permettant de comprendre à la fois la situation de travail et la situation d'intervention. Les connaissances mobilisées sont particulièrement explicitées par Girardot et Forrierre. Il s'agit :

- De connaissances sur les liens organisation-santé-performance et le modèle de l'humain au travail : p.ex. modèle plurifactoriel de la survenue de TMS, écart prescrit-réel, variabilités, régulations, compromis opératoire, déterminants de l'activité...

- De connaissances spécifiques liées à la situation d'intervention (p. ex. la posture de l'usager : prescripteur / coopérant (Falzon \& Lapeyrière, 1998) ou les différents pôles de la relation de service (Caroly \& Weill-Fassina, 2004) dans le cas de Girardot et Forrierre ;

De connaissances sur la démarche en elle-même: la conduite d'un projet de conception d'une situation de travail, les constructions de démarche de prévention durable (Daniellou, Caroly, Coutarel, Escriva, Roquelaure, Schweitzer, 2008 cité par Girardot et Forrierre, ce dossier).

31 Ces éléments sont abordés en se basant sur les expériences et les éléments des terrains en question « de manière concrète et interactive » (ibid., p. 12), ces expériences étant amenées de manière planifiée et d'autres évoquées de manière opportune.

D'autre part, les dispositifs s'appuient sur la mise en visibilité du travail et le développement d'un autre regard sur le travail, à travers la construction collaborative d'un diagnostic qui permet aux participants de développer des compétences « analytiques collectives ", c'est-à-dire construire une intelligibilité mutuelle des situations ; une vision holistique des organisations; et une représentation de l'interdépendance entre acteurs et de la diversité des logiques et points de vue en présence (Carta \& Falzon, ce dossier)

Pour Girardot et Forrierre, il s'agit de la construction collaborative d'un diagnostic à partir d'éléments recueillis par les opérateur.trice.s sur le terrain, puis discutés et analysés dans un "groupe de travail». Ces éléments recueillis (photos, récits d'évènements...) constituent les objets intermédiaires permettant de mettre en visibilité le travail et de l'analyser en mobilisant les cadres de l'ergonomie de l'activité. Girardot et Forrierre soulignent que ce diagnostic est selon eux " plus riche et plus près du réel », plus facilement appropriable que dans une intervention sans dispositif de formation et qu'ils favorisent la construction des pistes d'action.

Pour Carta et Falzon, plusieurs diagnostics organisationnels sont co-construits. Le premier permet aux participant.e.s de co-analyser le processus de maintenance dans lesquels il.elle.s sont engagé.e.s et de co-élaborer une représentation commune de ce processus de maintenance, ses contradictions et ses ressources. Il s'agit de construire une 
intelligibilité mutuelle des différentes logiques d'activités au sein de ce processus et de leurs impacts les unes sur les autres à travers la construction d'une cartographie de ce processus. Les participant.e.s ont ainsi pu identifier des «classes de problèmes prioritaires » et des « cas exemplaires » de ces problèmes. Cette co-construction repose là aussi sur la mobilisation d'objets intermédiaires (construits par l'ergonome sur la base d'un diagnostic initial), que les participant.e.s s'approprient au fil de la démarche. Selon les auteur.e.s cette co-élaboration a favorisé l'apprentissage des participant.e.s, et le développement de la «compétence analytique collective » (Carta et Falzon, ce dossier) et ceci sur un artefact particulier - une organisation - qui est devenue un " objet manipulable " pour eux. Ces apprentissages ont été soutenus au fil de la démarche: l'ergonome identifiait les besoins d'apprentissage à satisfaire et transformait alors les situations créées dans les espaces de discussion pour satisfaire ces besoins. Ainsi, un second diagnostic a porté sur la démarche mise en place par l'ergonome, à travers des séances de briefing et débriefing suite aux situations mises en place par l'ergonome, qui a conduit au développement de compétences à conduire un changement de manière située, participative et collaborative; et ceci de manière durable. Nous le verrons ce second diagnostic pose les bases du dispositif de pérennisation mis en place.

On voit donc que le diagnostic n'est plus seulement un objet intermédiaire de l'intervention, mais sa co-élaboration devient un moyen de "transformer le regard sur le travail » (Casse \& Caroly, ce dossier) et de développer des compétences de compréhension et d'analyse de situations de travail vécues.

\subsection{Dispositifs de mise en débats du travail : développement des collectifs et genèse du développement de l'organisation}

Trois des interventions capacitantes (Bonnefond ; Casse \& Caroly ; Rocha et al., ce dossier) mobilisent des dispositifs de type « espace de débats sur le travail ». Comme le rappellent Arnoud et Perez Toralla en introduction, ces dispositifs :

- visent à mettre en débat le travail autour de conflits de critères de qualité du travail (Clot, 2010), de conflits de normes (Davezies, 2012), de difficultés et tensions (Detchessahar, Gentil, Grévin, \& Stimer, 2012) ; ou de situations non anticipées (Casse \& Caroly ; Rocha et al., ce dossier);

- ont pour objectif de devenir des dispositifs durables, c'est-à-dire instutitionalisés et protégés au sein d'une organisation (Arnoud \& Perez Toralla, ce dossier);

- traversent tous les niveaux hiérarchiques d'une organisation.

37 Les dispositifs "espaces de débats sur le travail» tels qu'ils sont travaillés par l'ergonomie ou la psychologie du travail articulent des objectifs productifs - visant des transformations propres au contexte des interventions (p. ex. reconcevoir un dispositif de REX pour Casse et Caroly, ce dossier) - et constructifs, en lien avec le développement de collectifs de travail (cf.infra). Là encore, "analyse et action" sont indissociables (Rocha et al., ce dossier). Comme dans le cas précédent, ces dispositifs sont intégrés à des interventions "classiques » et s'appuient, au moins pour deux d'entre elles (Casse \& Caroly; Rocha et al., ce dossier), sur un diagnostic ergonomique construit par l'ergonome et préalable à la conception des espaces de débats. Ils s'appuient, par ailleurs, sur des méthodes permettant d'outiller les débats dans les espaces (p. ex. auto-confrontations croisées et montage vidéo pour Bonnefond ; simulations d'évènements significatifs pour Casse et Caroly). Enfin, ces dispositifs posent les bases des objectifs autopoïétiques, 
puisque les premières expérimentations des espaces de débats sur le travail constituent les prémisses des dispositifs de pérennisation, c'est-à-dire qu'ils font office de «laboratoires » pour initier le développement organisationnel (Detchessehar, cité dans Casse \& Caroly ; Carta \& Falzon, ce dossier) (cf. infra).

Les objectifs constructifs de la mise en place des espaces de débats sur le travail visant le développement des collectifs de travail (Caroly, 2010; Caroly \& Barcellini, 2013) soutiennent : la mise en visibilité d'une diversité de façons de faire et d'interpréter des situations de travail entre opérateur.trice.s, la conduite d'« analyses poussées sur le travail bien fait, ses problèmes, ses possibilités» (Bonnefond, ce dossier), la mise en débat argumentée des critères sous-jacents à la gestion de ces situations (p.ex. critères de qualité, critères d'appréciation du risque), afin « d'élargir le champ des représentations » des particpant.e.s (Casse \& Caroly, ce dossier), et de développer « de nouvelles façons de penser et d'agir", "des ressources collectives" (Bonnefond, ce dossier) et de résoudre des problèmes (Rocha et al., ce dossier). Ces ressources peuvent être réinvesties individuellement par les opérateur.trice.s (Bonnefond; Rocha et al., ce dossier). Les espaces de débats sur le travail sont donc également des espaces d'apprentissage individuel, même si c'est principalement leur fonction de développement des collectifs qui sera abordée ici.

Les ressources et les compétences collectives qui y sont développées sont diverses. Pour Casse et Caroly ou Rocha et al., ce sont des compétences collectives qui visent la gestion de la sécurité 6 : la compréhension d'évènements passés et le développement de règles d'action pour y faire face. Il s'agit également de compétences collectives à comprendre et estimer le risque encouru dans telle ou telle situation, à prendre la mesure des différences d'interprétation potentielles de ce risque, mais aussi à concevoir des solutions et à se projeter vers des situations futures. L'usage de la simulation apparaît alors comme une ressource, car elle "fait lien entre passé, présent et futur» (Casse \& Caroly, ce dossier) et permet d'envisager des «faires autrement». Les espaces de débats sur le travail permettent donc, comme la co-construction de diagnostic, de développer des compétences analytiques collectives, mais également des compétences à co-concevoir. Enfin, les espaces de débats permettent également de développer soutien, entraide et confiance au sein du collectif et intégration des novices (Casse \& Caroly; Rocha et al., ce dossier).

Pour finir sur ces objectifs constructifs, les espaces de débats sur le travail mis en place contribuent à transformer le "dialogue» entre opérateur.trice.s et hiérarchie, à redonner de l'autonomie à l'encadrement de proximité et aux opérateur.trice.s, en expérimentant un dialogue de qualité sur la qualité du travail (Bonnefond; Rocha et al., ce dossier). Rocha et al. montrent que les espaces de débats ont contribué à « ré-instaurer un dialogue entre encadrement et techniciens " sur la gestion des risques, et à proposer un dispositif organisationnel de "traitement» de ces risques - une transformation organisationnelle - dans lequel l'encadrement de proximité a un rôle clé (cf. infra). Le développement du collectif et de la relation à la hiérarchie devient une ressource pour initier une transformation d'une organisation du travail qui soit basée sur la confrontation de points de vue sur le réel, les situations de travail quotidiennes (Bonnefond, ce dossier). Transformation qui sera poursuivie au travers des dispositifs de pérennisation mis en place.

41 Ces objectifs constructifs sont soutenus par la construction d'un cadre participatif au sein des espaces de débats sur le travail. La construction du cadre est ici essentielle, car elle 
fixe : les méthodes permettant de mettre en discussion le travail réel et de concevoir des modalités d'actions, les règles fondamentales de la discussion (droit à l'erreur, devoir d'écoute, droit à la parole pour tou.te.s, devoir de communiquer (Detchessahar (2013), le rôle de la hiérarchie de proximité dans la conduite des espaces et dans le suivi des actions à leur niveau ou aux niveaux hiérarchiques supérieurs. Les espaces de débats sur le travail se doivent d'instaurer " un débat régulier sur le travail réel, finalisé par la recherche de solutions et animé et garanti par le manager de proximité » (Rocha et al., ce dossier). Le rôle du manager est essentiel notamment pour suivre le devenir des actions de transformations envisagées (cf. infra).

En fonction des interventions les cadres de proximité peuvent être présents ou non dès la mise en place des espaces de débats. Dans l'intervention de Bonnefond, ce sont les opérateur.trice.s seul.e.s qui ont produit des éléments de mis en visibilité du travail (un montage vidéo produit par les participants) qui ont dans un deuxième temps été mis en discussion dans un groupe incluant le management. Pour l'auteur, cette phase préalable est nécessaire pour assurer la construction d'un dialogue de qualité, car elle permet aux opérateur.trice.s de prendre la mesure du dispositif et de co-construire ce qu'il.elle.s veulent donner à voir de leur travail. Ceci permet d'éviter selon l'auteur, que les premières interactions autour du travail ne soient " parasitées " par « les rapports sociaux habituels", et par l'écart entre les critères managériaux de performance et ceux opérationnels, qui pourraient amener le management à « devoir refouler le dialogue, car les solutions (pourraient) dégrader » la performance théorique (Bonnefond, ce dossier).

Cependant, que les managers soient associés d'emblée ou non, il est clair que leur participation à la démarche va crescendo dans les interventions notamment en lien avec la pérennisation de la démarche. Ceci suppose néanmoins que les managers disposent eux.elles-mêmes d'un espace de débats sur le travail au niveau supérieur (Detchessahar, 2013 ; Rocha et al., ce dossier).

\section{Concevoir et intégrer des dispositifs de pérennisation de l'action : développement de la capacité des organisations à se transformer de manière durable et développement de l'activité de management}

Les dispositifs présentés dans la section précédente font office d'expérimentation - de laboratoires - pour penser différemment la relation santé-travail, la gestion des risques, la conduite du changement organisationnel. Ils conduisent à proposer des nouvelles organisations du travail, soit parce que ces dispositifs sont en soit des prototypes de ces nouvelles organisations qu'il s'agira ensuite d'ajuster, soit parce que l'issue de ces dispositifs est de concevoir ces nouvelles organisations. Comment alors, l'intervention peut-elle dépasser "l'ici et le maintenant » de ces expérimentations et favoriser leur maintien dans le temps, et plus largement la capacité des organisations de se transformer en prenant en compte le travail.

Ainsi, une des spécificités majeures des interventions ergonomiques capacitantes est qu'elles portent en elles les possibilités de leurs propres dépassements. En effet, l'objectif de développement des organisations implique d'aider ces organisations à concevoir, à 
mettre en usage et à faire vivre des dispositifs qui leur permettent de se transformer. Ceci transforme donc de manière majeure les dispositifs, voire la temporalité des interventions, puisqu'elles doivent contenir des dispositifs de pérennisation.

Dans les deux sections précédentes, nous avons mis en évidence des dispositifs qui soutiennent le développement des individus et des collectifs. Ces dispositifs constituent la genèse du développement organisationnel, mais ils ne suffisent pas en eux-mêmes, et doivent y être ajoutés des dispositifs de généralisation tout d'abord, puis de pérennisation. Toutes les interventions présentées dans ce numéro se sont dotées de ce type de dispositifs.

Un premier élément des dispositifs de pérennisation renvoie à la construction sociale de l'intervention et l'appui sur des instances, mises en place dès le début de l'intervention ou ad hoc (cf. section 2.1). Cet élément est très lié à la faisabilité de l'étape de généralisation décrite plus bas. Ces instances associent progressivement toute la ligne managériale aux dispositifs, avec des objectifs propres aux fonctions managériales associées, selon le principe de subsidiarité discuté par Rocha et al., Bonnefond ou Casse et Caroly, par exemple, qui décrivent le travail de ces instances et leurs articulations.

Un deuxième élément du dispositif est bien sûr la conception et à l'expérimentation de méthodes et de dispositifs à un niveau local de l'organisation, voire la conception d'un prototype de démarches. C'est ce que nous avons décrit dans les sections précédentes.

Un troisième élément vise à déployer ce dispositif-prototype, ou celui qui est généré par l'étape d'expérimentation plus généralement de manière transverse et/ou verticale dans l'organisation et à l'ajuster. Carta et Falzon soulignent qu'il.elle.s ont mobilisé plus de 40 managers appartenant à 5 niveaux hiérarchiques, ainsi que tout l'encadrement de proximité, les managers des ressources humaines et des services d'hygiène et sécurité au travail. Bonnefond décrit le déploiement vertical de son dispositif d'intervention au sein de l'organisation: les critères de qualité du travail sont d'abord débattus au sein d'une instance au plus près du travail qui n'implique que des opérateur.trices. Ce groupe expérimente le dialogue sur le travail grâce aux autoconfrontations croisées et produisent des montages qu'il.elle.s livrent à un comité de suivi composé du management et des organisations syndicales, et ainsi de suite jusqu'à la direction de l'usine et puis du groupe. Les montages vidéos ont là deux fonctions essentielles : la mise en visibilité du réel du travail et un rôle d'exemple puisque le comité de suivi est invité à débattre tel que ses membres le voient faire dans les films. L'institutionnalisation du débat sur le travail traverse ainsi toute la ligne hiérarchique. L'intervention de Casse et Caroly s'appuie elle aussi sur le travail de plusieurs instances d'abord au plus près du travail de production puis remontant dans la ligne hiérarchique, avec un équipement méthodologique qui varie là aussi suivant ces groupes : des premiers ateliers de travail réflexif sur des évènements significatifs avec quelques opérateurs, jusqu'à la généralisation du dispositif à tous les opérationnels d'un même métier (déploiement transverse et vertical), puis à plusieurs groupes inter-métiers différents impliqués dans la gestion des risques à travers la méthode de simulation d'évènements passés (déploiement transverse et vertical). L'effacement de l'ergonome est progressif puisque c'est le chef d'équipe qui animait ces dernières sessions, annonçant la phase de pérennisation.

50 À ce processus de généralisation, Rocha et al. ajoutent un processus d'ancrage rapide dans l'organisation puisque parallèlement à la mise en place des espaces de débats sur le travail, les opérateur.trice.s conçoivent avec l'ergonome un dispositif quotidien de mise en débat ancré dans le dispositif organisationnel existant (p. ex réunions existantes). 
51 Un quatrième élément vise à mettre en place les conditions de pérennisation, qui passe par une inscription organisationnelle des dispositifs. L'ancrage progressif dans l'organisation - c'est-à-dire le passage de la généralisation à la pérennisation - implique :

- le développement des compétences individuelles et collectives (cf. supra) ;

- le développement par le management de compétences relatives à la conduite de projets organisationnels située et effectivement participative (Barcellini, 2015), c'est-à-dire la mise en place: (1) de conduite de projet située et non l'application de règles standards de conduite de projet sans réflexion sur les spécificités et le contexte du projet en question ; (2) de situations réellement collaboratives au sein de ces projets. Le développement de ces compétences implique que l'intervention ergonomique capacitante soutienne la définition de critères de qualité quant à la conduite de démarche collaborative de conception organisationnelle. C'est le développement de ces compétences qui est à la base du développement organisationnel.

- un effacement progressif de l'ergonome au profit du management pour concevoir et animer les instances constituées. Ceci passe par le développement de l'activité de management (cf. infra).

- la co-conception et l'adaptation du dispositif à pérenniser. Bonnefond ou Rocha et al., décrivent par exemple que l'espace de débats sur le travail s'est transformé en un prototype co-conçu avec certains membres du groupe (p.ex. le prototype de «Dialogue sur la Qualité du Travail - DQT a été conçu par un chef d'atelier, quatre référents, deux chefs d'équipes et les intervenants);

- l'inscription dans l'organisation du dispositif et des référents qui le portent, et le maintiendront (Bonnefond; Girardot \& Forrierre ; Casse \& Caroly, ce dossier). Bonnefond illustre comment cette fonction de référent a émergé au fil de l'intervention et à la demande des participant.e.s, pour finalement être inscrite dans l'organisation. Cette fonction est essentielle pour garantir la pérennité du dispositif: le contrôler en s'assurant de la " cohérence entre décision et action", le maintenir et agir comme "force de rappel " auprès du management, et « hiérarchiser les problèmes à traiter » (Bonnefond, ce dossier). Cette fonction est légitime aux yeux du collectif et légitimé par lui du fait de son émergence de la démarche mise en place, c'est-à-dire de l'implication historique des référents.e.s qu'il.elle.s soient élu.e.s ou choisi.e.s par les participant.e.s. (Bonnefond; Girardot \& Forriere, ce dossier). À cette fonction de référent peut s'ajouter la construction d'instances garantes de la pérennité et inscrites dans la structure organisationnelle (telle la "commission DQT » impliquant direction et organisation syndicale décrite par Bonnefond, ce dossier). L'inscription organisationnelle des dispositifs est essentielle pour prévenir «l'amnésie » du dispositif et construire la mémoire des actions mises en place, il faut donc en garder trace (Bonnefond; Carta \& Falzon, ce dossier). Cet ancrage concerne :

- la mise en place de formation à destination du management " garant de la durabilité » (Carta \& Falzon, ce dossier) ;

- la transformation des règles de l'organisation (p.ex. une remontée quotidienne des évènements en lien avec la sécurité chez Rocha et al.) ;

- l'inscription dans des référentiels ou documents de l'organisation (p.ex. référentiel quant à la conduite de projets organisationnels chez Carta et Falzon; document formalisant « l'organisation du retour d'expérience chez les exploitants de tunnels routiers » pour Casse et Caroly) ;

- jusqu'à l'inscription de la participation aux dispositifs dans la grille salariale (p.ex. pour la participation aux chantiers « Dialogue sur la Qualité du Travail » de Bonnefond). 
- La mobilisation de dispositifs analogues pour répondre à d'autres questions de l'organisation. Carta et Falzon ont observé que la déclinaison du dispositif de formation aux garants de la durabilité a été déployée à d'autres secteurs et que plusieurs démarches ont été lancées et pilotées à l'aide des référentiels produits, sous forme de formations à d'autres acteurs de l'entreprise (Carta \& Falzon, ce dossier)

Pour finir, ajoutons que tou.te.s les intervenant.e.s soulignent le rôle important du management et le développement de l'activité de management tout au long de leurs interventions. Ceci est particulièrement prégnant dans l'intervention de Rocha et al., qui identifiait en amont de l'intervention le « silence organisationnel » qui avait pris place au sein de l'organisation, révélateur d'une « rupture de dialogue » entre les opérateur.trice.s de production et le management. Un objectif de leur intervention était donc de reconstruire ces possibilités de dialogue. Or, ceci ne va pas de soi, car les objectifs du management peuvent être en contradiction avec ceux des opétateur.trice.s de production ce qui peut entraver la mise en place du débat, sans précaution de l'intervenant. C'est notamment pour se prévenir de ce risque d'entrave au dialogue que Bonnefond choisit de ne pas associer le management de proximité aux espaces de débats fondateurs de la démarche auprès des opérateur.trice.s.

Le développement de cette activité est soutenu par l'expérimentation par le management de la conduite de la démarche ou de certaines étapes : conception et conduite d'espaces de débats chez Casse et Caroly, ou le management de la démarche chez Carta et Falzon, ou encore la prise en charge des remontées des situations de travail dans le dispositif quotidien mis place chez Rocha et al.. Cette expérimentation peut être soutenue, ou non, par un acte de formation intentionnel de l'intervenant, tel que Carta et Falzon qui conduisent des formations, planifiées ou opportunistes, auprès d'acteur.trice.s identifié.e.s comme des garant.e.s de la démarche. Il s'agit en fait de soutenir la capacité du management à produire du sens autour de leurs actions (cf. supra). Carta et Falzon développent particulièrement cet aspect en se référant au processus de sensemaking. Il convient néanmoins que le management soit en mesure de traiter "ses vulnérabilités ", de prendre conscience des ressources et faiblesses organisationnelles (Rocha et al., ce dossier).

Ce développement concerne notamment la capacité des managers à "gérer " les situations qui leurs sont remontées, à identifier les «bons interlocuteurs » et à suivre les actions identifiées et informer sur les possibilités de réalisation, ou non, et l'avancée des actions identifiées. Il s'agit ici de garder une convergence entre "discours et action». Ceci est essentiel pour maintenir la confiance dans l'efficacité des dispositifs conçus, et donc sa pérennité (Rocha et al., ce dossier). Plus largement, Rocha et al. renvoient ce développement à celui du principe de subsidiarité, d'autonomie et de pouvoir d'agir dans les organisations : «quel est le niveau pertinent le plus bas pour gérer la situation discutée? Que doit faire le niveau supérieur pour donner du pouvoir d'agir au niveau inférieur afin que ce dernier ait les ressources suffisantes pour traiter les questions remontées?»

\section{Discussion et perspectives}

55 Les interventions relatées dans ce numéro spécial permettent de progresser dans la modélisation de ce que peuvent être des interventions ergonomiques capacitantes : qui 
articulent aux dispositifs d'intervention, des dispositifs de formation-apprentissages, d'expérimentations de méthodes d'actions développementales et de pérennisation.

Ce numéro spécial ouvre également des pistes de formation et de recherche relatives : à la transformation des activités de l'ergonome au sein des ces interventions capacitantes, à la poursuite de la production de connaissances sur ces interventions et au développement de la discipline.

\subsection{Vers une transformation des activités de l'ergonome?}

Plusieurs auteur.e.s mettent en évidence le rôle et les activités qui peuvent être mises en œuvre par l'ergonome - ou par l'intervenant - conduisant des interventions ergonomiques capacitantes (Carta \& Falzon; Girardot \& Forrierre; Bonnefond, ce dossier) :

- L'ergonome-stratège politique qui se positionne, négocie et implante son intervention et l'ajuste en fonction de sa lecture de l'évolution de l'intervention, du positionnement des acteurs organisationnels et de leur rapport de pouvoir (p. ex. Noyer \& Barcellini, 2014). Ceci implique de reconcevoir « dans l'usage les moyens, les structures et les ressources mobilisées » par l'intervention (Carta \& Falzon, ce dossier) et de disposer de compétences «de négociation, de traduction du discours ergonomique en langage "politique-stratégique», mais également de « marketing » de l'ergonomie.» (Carta \& Falzon, ce dossier) ;

- L'ergonome-» praticien réflexif » qui ajuste son action, notamment en lien avec la poursuite des objectifs constructifs, productifs et autopoïetiques ;

- L'ergonome-formateur organisant les apprentissages individuels, collectifs et organisationnels, qui étaye les participant.e.s, les tutore pour les amener « à construire les ressources et les solutions nécessaires au développement de l'organisation " (Carta \& Falzon, ce dossier), et ceci en s'appuyant sur le travail réel et les expériences vécues par les particpant.e.s. ;

- L'ergonome-concepteur: d'espaces-temps outillés d'objets intermédiaires et de cas représentant le travail, ces espaces-temps outillés étant constitutifs de dispositifs de formation-apprentissage, d'expérimentation, de pérennisation ;

- L'ergonome-manager des dispositifs mis en place : à la fois au niveau de la structure du processus, pour garantir sa cohérence et son avancée (Carta \& Falzon, ce dossier), qu'au niveau plus fin des interactions entre acteurs, dans les espaces-temps qu'il.elle créé. L'ergonome est le garant du cadre participatif posé (p. ex. Cahour, 2002 ; Bonnefond, ce dossier) : il.elle assure la "démocratie de l'espace de parole», une "dynamique collaborative transverse aux strates hiérarchiques » (Carta \& Falzon, ce dossier), et « d'un primat du réel » dans les interactions, notamment face aux rapports sociaux installés ou aux relations de subordination (Bonnefond, ce dossier).

Si la discipline veut assumer pleinement ce nouveau positionnement développemental, se pose alors la question du développement de ces rôles et des activités qui leur sont associés, dès la formation initiale ou en formation continue. Certains de ces rôles sont déjà travaillés en formation initiale, notamment les activités en lien avec le rôle de "praticien réflexif», qui a profondément nourri les dispositifs de formation à l'intervention (p. ex. Falzon \& Barcellini, 2014; Teiger \& Lacomblez, 2013). De même, le rôle d'ergonome-stratège est également travaillé dans les enseignements, notamment au niveau de l'analyse stratégique de la demande, il pourrait néanmoins être renforcé à travers des enseignements relatifs à la lecture stratégique et politique des organisations 
et des relations de pouvoir qui s'y jouent. La lecture des interventions présentées souligne le besoin de renforcer les capacités formatrices des ergonomes et de " chefs d'orchestre » et concepteurs d'une diversité de dispositifs d'intervention, qui dépassent - bien qu'elle ne les ignore pas - la construction d'un diagnostic ou la mise en place de simulations. Pour ce qui est des capacités formatrices, un renforcement de la présence des enseignements en didactique professionnelle semble essentiel. Pour ce qui est du renforcement des compétences de conception d'une diversité de dispositifs, la documentation des expériences d'interventions concrètes, et des « innovations » qu'elles intègrent - mises en œuvre par divers praticiens et praticiennes (chercheur.e, enseignant.e-chercheur.e, consultant.es, ergonomes internes) est une ressource indispensable : que cela soit dans des numéros spéciaux ou au sein de congrès (p. ex. la SELF) ou de journée sur la pratique (p.ex. Journée de Bordeaux). Dans ce sens, des expériences de mises en récit construites entre enseignant.e-chercheur.e (p. ex. Noyer \& Barcellini, 2014 ; Girardot \& Forrierre, ce dossier) apparaissent prometteuses, malgré les limites réelles soulignées par Arnoud et Perez Toralla et qu'il faudrait chercher à dépasser. Au-delà de ces expériences de mises en récit, il est nécessaire de progresser sur la modélisation de ces interventions pour pouvoir les réinvestir dans les enseignements, comme nous l'explicitons dans la section suivante.

\subsection{Développement de la discipline} expériences d'interventions capacitantes mises en œuvre à d'autres échelles ou dans d'autres secteurs. C'est le cas notamment de deux thèses en cours dont nous avons connaissance, mais il en existe sans doute d'autres. La thèse de Thomas s'intéresse au développement d'un collectif transverse en charge de la fabrique de la prescription dans une industrie ultra-sûre et de la mise en place d'un dispositif permettant de pérenniser le développement de ce collectif (Thomas, Barcellini, Quatrain, Ricard, \& Falzon, 2015). La thèse de Chizallet (Chizallet, Prost, \& Barcellini, soumis) s'intéresse au développement d'une intervention capacitante en conduite du changement dans le cadre de l'accompagnement des transformations du travail d'agriculteur.trice.s engagé.e.s dans des transitions agro-écologiques. Dans ce cadre, l'intervention a pour objectif de développer l'activité de concepteur des agriculteur.trice.s (Prost, Prost, \& Cerf, à paraître) - vue comme une ressource pour assurer la pérennité de ces transitions et des conditions de travail soutenables pour les agriculteurs - et ceci à travers la conception d'un dispositif déployable à large échelle au sein d'un territoire. l'œuvre au sein de ces divers dispositifs et ceci dans une perspective longitudinale, tel que proposé dans Barcellini (2015). Par exemple, quelle est l'activité de l'ergonome dans les espaces de débats sur le travail, comment évolue-t-elle au fil de l'intervention? Quelles sont les activités du management et des autres participant.e.s? Quel rôle des objets intermédiaires et de la situation d'interactions conçues vis-à-vis des apprentissages ? Sur quels indices l'ergonome re-oriente-il.elle son intervention? Quelle est l'implantation stratégique et politique des interventions et comment évolue-t-elle ? La réponse plus fine à ces questions est essentielle. 
Une troisième perspective tient à la poursuite de recherches en ergonomie déjà entreprises :

- Sur l'activité du management (de proximité au top management) (p. ex. Piney, 2015). Cette perspective obéit au même mouvement qui a vu au milieu des années 80 une influence des travaux sur les activités de conception sur le développement des démarches d'intervention (p.ex. Barcellini, 2015 ; Daniellou, 1992 ; Falzon, 1993 ; Garriguou, 1992) ;

- Ou sur les propriétés capacitantes, ou non, des organisations du travail. Produire des connaissances sur le réel d'organisations du travail plus capacitantes (p. ex. celle des communautés en ligne, Barcellini, 2015) peut être un moyen de mettre en discussion les formes actuelles d'organisation du travail (lean conception, néo-taylorienne, organisation mondialisée, sous-traitance, etc.), qui contraignent fortement le travail des salariés dans les entreprises (intensification, pression temporelle, relation de dépendance, etc.).

Ces deux points militent pour un rapprochement, déjà en cours, avec les sciences de gestion et de l'organisation ou la sociologie des organisations.

Une quatrième perspective tient à la diffusion des apports de ces travaux auprès des entreprises, auprès d'autres disciplines, et au niveau international. En effet, l'ergonomie n'est pas encore totalement identifiée - voire pas du tout - comme une discipline contributrice aux démarches de changement organisationnel dans les entreprises, qui restent l'apanage des sciences de gestion et de l'organisation. Or, ces disciplines sont elles aussi porteuses actuellement d'un courant «constructif» qui milite pour le développement de démarches de conduite $d u$ changement qui s'appuie sur l'apprentissage des «acteurs " pour développer la capacité organisationnelle à changer (Soparnot, 2008). Dans ce cadre, les propositions de l'ergonomie constructive permettent d'opérationnaliser cette intention, et méritent d'être publiées dans les revues de ces disciplines. Par ailleurs, il s'agit également de chercher à convaincre décideurs, prescripteurs, syndicats et acteurs politiques de l'intérêt d'une réelle prise en compte d'une vision capacitante du travail et de l'organisation, dans les démarches de changements (Daniellou, 2013). Une stratégie proposée par la discipline est de «faire la preuve par le local» (op.cit., p.7), par la mise en œuvre effective d'interventions, permettant aux organisations d'expérimenter « dans leurs corps » les potentialités de ces interventions et à l'encadrement de prendre conscience de son rôle pour favoriser la mise en discussion du travail dans la conduite du changement et ceci à des niveaux de l'organisation pertinents (p. ex. Daniellou, 2013 ; Rocha, 2014).

Enfin, le développement des interventions ergonomiques capacitantes passe également par une diffusion internationale de l'approche du travail et de ses transformations, telle qu'elle est pensée par l'ergonomie de l'activité et l'ergonomie constructive. Plusieurs initiatives ont été récemment menées dans ce sens, comme la parution de l'ouvrage Constructive Ergonomics coordonné par Pierre Falzon en 2013, le projet d'organisation d'un symposium sur ce thème au prochain congrès de l'International Ergonomics Association (Arnoud, Barcellini, Carta, \& Perez Toralla, soumis). 


\section{BIBLIOGRAPHIE}

Andriessen, J., Baker, M., \& Suthers, D. (2003). Arguing to Learn: Confronting cognitions in computer supported argumentation. Dordrecht, The Netherlands : Kluwer.

Arnoud, J. (2013). Conception organisationnelle : pour des interventions capacitantes. Thèse de doctorat en ergonomie, Cnam, février.

Arnoud, J., Barcellini, F., Carta, G., \& Perez Toralla, M.S (soumis). Constructive ergonomics: eabling intervention and related methodological issues. Symposium submitted to IEA 2018, Florence, Italy

Arnoud, J., \& Falzon, P. (2013). Changement organisationnel et reconception de l'organisation : des ressources aux capabilités. Activités, 10(2), 109-130. URL : https://activites.revues.org/760

Arnoud, J., \& Falzon, P. (2017). Intervenir « sur » l'organisation : vers un cadre méthodologique. In A.-L. Ulmann, A. Weill-Fassina, \& H. Benchekroun (Eds.), Intervenir - Histoires, recherches, pratiques. Toulouse : Octarès.

Baker, M. (2004). Recherche sur l'élaboration de connaissances dans le dialogue. Habilitation à Diriger des Recherches, Université de Nancy 2.

Baker, M. (2009). Argumentative interactions and the social construction of knowledge. In N.M. Mirza \& A.-N. Perret-Clermont (Eds.) Argumentation and Education: Theoretical Foundations and Practices (pp. 127-144). Berlin : Springer Verlag.

Barcellini, F. (2015). Développer des Interventions Capacitantes en Conduite du Changement : comprendre le travail collectif de conception, agir sur la conception collectice du travail. Habilitation à Diriger des Recherches, Université de Bordeaux, 17 avril.

Barcellini, F., Van Belleghem, L., \& Daniellou, F. (2013). Les projets de conception comme opportunité de développement des activités. In P. Falzon (Ed.). Ergonomie Constructive (pp. 191-206). Paris, France : PUF.

Béguin, P. (2003). Design as a mutual learning process between user and designers. Interacting with Computer, 15(5), 709-730.

Bourgeois, F. (2012). Que fait l'ergonomie que le lean ne sait / ne veut pas voir ? Activités, 9(2), 138-147. URL : https://activites.revues.org/444

Cahour, B. (2002). Décalages socio-cognitifs en réunion de conception participative. Le Travail Humain, 65(4), 315-337.

Caroly, S. (2010). L'activité collective et la réélaboration des règles : des enjeux pour la santé au travail. Habilitation à Diriger des Recherches, Université de Bordeaux, 3 mars 2010

Caroly, S., \& Barcellini, F. (2013). Le développement de l'activité collective. In P. Falzon (Ed.)

Ergonomie Constructive (pp. 33-46). Paris, France : PUF.

Caroly, S., \& Weill-Fassina, A. (2004). Évolutions des régulations de situations critiques au cours de la vie professionnelle dans les relations de service. Le Travail Humain, 67(4), 305-332.

Carta, G., Falzon, P., \& Re, A. (2015). A constructive and participative approach to the analysis and redesign of the organization. 19th Triennial Congress of the International Ergonomics Association.

Melbourne, 9-14 August. 
Chizallet, M., Prost, L., \& Barcellini, F. (soumis). Conduire une transition agro-écologique de manière autonome et durable : Re-penser le travail passé pour concevoir le travail futur. Psychologie française.

Clot, Y. (2010). Le travail à cœur : Pour en finir avec les risques psychosociaux. Paris : La Découverte. Coutarel, F., \& Petit, J. (2009). Le réseau social dans l'intervention ergonomique : enjeux pour la conception organisationnel. Management et Avenir, 27(7), 135-151.

Daniellou, F. (1992). Le statut de la pratique et des connaissances dans l'intervention ergonomique de conception. Habilitation à Diriger des Recherches, Université Victor Segalen Bordeaux 2.

Daniellou, F. (2013). Le travail bien fait contre l'organisation : enjeux de santé et de production. In L. Lerouge (Ed.) Approche interdisciplinaire des risques psycho-sociaux. Toulouse, France : Ocatrès. Daniellou, F. (Ed.), Caroly, S., Coutarel, F., Escriva, E., Roquelaure, Y., \& Schweitzer, J-M, (2008). La prévention durable des TMS. Quels freins? Quels leviers d'action? Recherche-action 2004-2007. Rapport de recherche pour Direction générale du travail. http://www.anact.fr/

Davezies P. (2012), Dimensions collectives du travail : les enseignements de la souffrance au travail. Communication présentée lors du Séminaire interne 2011-2012, Ergonomie et société : enjeux et perspectives, Paris, Cnam.

Delgoulet, C., \& Vidal-Gomel, C. (2013). Le développement des compétences : une condition pour la construction de la santé et de la performance au travail. In P. Falzon (Ed.), Ergonomie constructive (pp. 19-33), Paris : PUF.

Detchessahar, M. (2013). Faire face aux risques psycho-sociaux : quelques éléments d'un management par la discussion. Négociations, 19(1), 57-80.

Detchessahar, M., Gentil, S., Grévin, A., \& Stimec, A. (2012) Le design de la recherche-intervention en management : réflexions méthodologiques à partir d'une intervention dans une clinique. XVII ${ }^{\mathrm{e}}$ Congrès de l'AGRH, Nancy, France.

Doise W., \& Mugny, G. (1981). Le développement social de l'intelligence. Paris : Interéditions. Teiger \& Lacomblez, 2013

Dugué, B., Petit, J., \& Daniellou, F. (2010). L'intervention ergonomique comme acte pédagogique. Pistes, 12(3).

Falzon, P. (1993) Médecin, pompier, concepteur : l'activité cognitive de l'ergonome. Performances Humaines et Techniques, 66, 35-45.

Falzon, P. (2013). Ergonomie Constructive. Paris, France : PUF.

Falzon, P., \& Barcellini, F. (2014). Former à la pratique en ergonomie- Texte de cadrage relatif à l'accompagnement du mémoire professionnel d'ergonomie. Cours «ERG 245 : Intervenir en ergonomie » du CNAM, octobre 2014.

Falzon, P., \& Lapeyrière, S. (1998). L'usager et l'opérateur : ergonomie et relations de service. Le Travail Humain, 61(1), 69-90.

Garrigou, A. (1992). Les apports des confrontations d'orientation socio-cognitives au sein de processus de conception participatifs : le rôle de l'ergonomie. Thèse de doctorat en ergonomie, CNAM.

Guérin, F., Laville, A., Daniellou, F., Duraffourg, J., \& Kerguelen, A. (1991 ; 2007). Comprendre le travail pour le transformer : la pratique de l'ergonomie. Lyon : ANACT.

Maline, J. (1994). Simuler le travail. Lyon : ANACT. 
Mollo, V., \& Nascimento, A. (2013). Pratiques réflexives et développement des collectifs, des individus et des organisations. In P. Falzon (Ed.). Ergonomie Constructive (pp. 207-222). Paris, France : PUF.

Muller Mirza, N., \& Perret-Clermont, A-N. (2009). Argumentation and Education. Dordrecht : Springer Verlag.Baker.

Nascimento, A., \& Falzon, P. (2009). Produire la santé, produire la sécurité. Activités, 6(2). URL : http://activites.revues.org/2225; DOI : 10.4000/activites.2225

Noyer, N., \& Barcellini, F. (2014). Construire un projet durable de prévention des TMS : dimensions stratégique et pédagogique d'une intervention ergonomique. Pistes, 16(3).

Pastré, P. (2005). Apprendre par la simulation: de l'analyse du travail aux apprentissages professionnels. Toulouse, France : Octarès.

Perez Torralla, M.S. (2013). Pour une prescription capacitante, ergonomie et débats des règles du travail : le cas d'une entreprise déployant la lean production. Thèse de doctorat en ergonomie, CNAM, 20 décembre 2013.

Petit, J. (2005). Organiser la continuité du service : intervention sur l'organisation d'une mutuelle de santé . Thèse de doctorat en ergonomie, Université de Bordeaux 2, 7 décembre 2005.

Piney, C. (2015). Transformation de l'activité d'encadrement de proximité : que fait le pilotage par la performance à la "proximité" des cadres ? : Le cas d'une grande administration publique. Thèse de doctorat en ergonomie du Conservatoire National des Arts et Metiers, $1^{\text {er }}$ décembre 2015.

Prost, M., Prost, L., \& Cerf, M. (à paraître). Les échanges virtuels entre agriculteurs : un soutien à leurs transitions professionnelles? Raisons éducatives

Raspaud, A. (2014). De la compréhension collective de l'activité réelle à la conception participative de l'organisation : plaidoyer pour une intervention ergonomique capacitante. Thèse de doctorat en ergonomie, CNAM. 10 décembre 2014.

Rocha, R. (2014). Du silence organisationnel au développement du débat structuré sur le travail : les effets sur la sécurité et sur l'organisation. Thèse de doctorat en ergonomie, Université de Bordeaux, 21 novembre 2014.

Saint-Vincent, M., Vézina, N., Bellemare, M., Denys, D., Ledoux, E., \& Imbeau, D. (2011). L'intervention en ergonomie. Québec : Éditions MultiMondes, IRSST

Schön, D. (1987). Educating the Reflective Practitioner (Vol. Jossey-Bass). San Francisco, CA.

Soparnot, R. (2008). Vers une gestion stratégique du changement : une perspective par la capacité organisationnelle de changement. Management et Avenir, 28, 104-122.

Teiger, C., \& Lacomblez, M. (2013). Se former pour transformer le travail. Dynamiques de constructions d'une analyse critique du travail. Laval, Canada : Presses de l'université de Laval.

Terssac, G. de (2003). La théorie de la régulation sociale de Jean-Daniel Reynaud - Débats et prolongements. Paris, France : La Découverte.

Thomas, C., Barcellini, F., Quatrain, Y, Ricard, B., \& Falzon, P. (2015). The production of prescriptive documents in safety-critical organizations: an exploratory diagnosis. In proceedings of IEA 2015, Melbourne, Australia.

Vinck, D. (2009). De l'objet intermédiaire à l'objet frontière : vers la prise en compte du travail d'équipement. Revue d'anthropologie des connaissances, 3(1), 51-72.

Vygotski., L. (1997). Pensée et Langage. Paris : Éditions La Dispute. 


\section{NOTES}

1. Le concept d'organisation renvoie ici à un ensemble de règles structurant le travail (horaires, organigramme, possibilités de coopération....), voir Arnoud (2013); Petit (2005); de Terssac (2003).

2. Pour une discussion plus approfondie des modèles organisationnels - organisation comme structure vs. organisation comme le fruit d'interaction - le lecteur intéressé pourra se référer à Petit (2005) et Arnoud (2013).

3. Le terme collaboratif est ici employé dans un sens «fort», il renvoie à la co-élaboration effective de connaissances à travers des interactions langagières (voir Barcellini, 2015 pour une synthèse).

4. Nous conserverons dans la suite le terme de formation-apprentissage car, si pour Carta et Falzon et Girardot et Forrierre (ce dossier), il s'agit de dispositifs de formation explicite, les dispositifs des autres interventions renvoient plutôt à des dispositifs d'apprentissage car elles ne précisent pas les modalités de formation employées.

5. Pour reprendre Girardot et Forrierre (ce dossier) «l'intervention ergonomique étant entendue ici comme l'intervention de transformation (Teiger \& Lacomblez, 2013) qui consiste pour un ergonome à mettre en cuvre la démarche d'analyse ergonomique du travail dans le but de transformer les situations de travail (Guérin, Laville, Daniellou, Duraffourg, \& Kerguelen, 1991/2007; St Vincent, Vézina, Bellemare, Denys, Ledoux, \& Imbeau, 2011). »

6. En référence à l'approche de la sécurité gérée (voir p. ex. Nascimento \& Falzon, 2009).

\section{AUTEUR}

\section{FLORE BARCELLINI}

Le CNAM, Centre de Recherche sur le Travail et le Développement, 41 rue Gay-Lussac, 75005

Paris. flore.barcellini@lecnam.net 